\title{
Individualized exercise and the health of the AYA cancer survivor population
}

Adam Corya ${ }^{1}$, Jacob Conroy ${ }^{2}$, Abigail Bolt ${ }^{3}$, Jessica Ricks ${ }^{4}$, Nicholas Kelly ${ }^{5}$, Danielle Halsey ${ }^{1}$, Tammy Sajdyk $^{1}$, Jamie Renbarger ${ }^{1}$

${ }^{1}$ Indiana University School of Medicine, Indianapolis, IN; ${ }^{2}$ Marian University School of Osteopathic Medicine, Indianapolis, IN; ${ }^{3}$ Vanderbilt University, Nashville, TN; ${ }^{4}$ Indianapolis Community Healthplex; ${ }^{5}$ Indiana University Purdue University Indianapolis School of Health and Human Sciences

\section{Background + Hypothesis}

Adolescent and young adult cancer survivors (AYACS: ages 15-39) have an $84.5 \%$ five-year survival rate. ${ }^{(1)}$ AYACS have a 10 times greater risk to develop cardiac disease compared to healthy peers. ${ }^{(2)}$ This is in part due to their lower physical activity. ${ }^{(3)}$ Structured exercise in adult cancer survivors improves strength, fatigue, VO2, and antioxidant levels and it decreases markers of cellular damage ${ }^{(4,5)}$. AYACS could benefit similarly, reducing long-term health effects. Although evidence suggests exercise is beneficial in older cancer survivors, this has not been demonstrated in AYACS. We hypothesized that a 12-week one-on-one multi-modal, community-based exercise program would improve AYACS outcomes compared to baseline or inactive AYACS. The current study hopes to demonstrate the feasability of an exercise intervention in a community setting within Indianapolis.

\section{Methods}

Six individuals were included in a feasibility trial for a larger pilot study of 374 participants. On day 1 , baseline assessments were performed for experimental outcomes: body composition, strength, flexibility, VO2peak, balance, plasma biomarkers, PA, psychological health, health-related quality of life, and fatigue. Mini reassessments were performed at week 5, measuring strength and VO2peak with an estimated 1-rep maximum and 6-minute Walk Test respectively; in the larger pilot study participants will be reassessed at weeks 12 and 24. Participants train for 60 minutes (20 cardio, 30 weights, 10 stretching) 3 times a week for 12 weeks, one-on-one with a cancer exercise specialist.

\section{Results}

The average change in VO2peak was $+25.3 \%$ and in strength was $+17.5 \%$ (no statistical analysis). Adherence was $90.9 \%$.

\section{Conclusion + Potential impact}

This trial suggests the feasibility of a pilot larger study. The greatest limitation was that the population sample was not within the AYACS age range. However, as the goal was to show feasibility rather than to prove efficacy, the sample gave useful information.

Sources (1-5) 
1. Anderson $C$, Smitherman AB, Nichols HB. Conditional relative survival among long-term survivors of adolescent and young adult cancers. Cancer. 2018;124(14):3037-43.

2. Armstrong GT, Kawashima T, Leisenring W, Stratton K, Stovall M, Hudson MM, et al. Aging and risk of severe, disabling, life-threatening, and fatal events in the childhood cancer survivor study. J Clin Oncol. 2014;32(12):1218-27.

3. Wu YP, Yi J, McClellan J, Kim J, Tian T, Grahmann B, et al. Barriers and Facilitators of Healthy Diet and Exercise Among Adolescent and Young Adult Cancer Survivors: Implications for Behavioral Interventions. J Adolesc Young Adult Oncol. 2015;4(4):184-91.

4. Repka CP, Hayward R. Oxidative Stress and Fitness Changes in Cancer Patients after Exercise Training. Med Sci Sports Exerc. 2016;48(4):607-14.

5. Repka CP, Hayward R. Effects of an Exercise Intervention on Cancer-Related Fatigue and Its Relationship to Markers of Oxidative Stress. Integr Cancer Ther. 2018;17(2):503-10. 